\title{
Mathematical modeling of temperature during laser forming using bimodal beam
}

\author{
Masoud Sistaninia $^{\mathrm{a}^{*}}$ and Mahjoubeh Sistaninia ${ }^{\mathrm{b}}$
}

${ }^{a}$ Materials Center Leoben Forschung GmbH, Roseggerstrasse 12, A-8700 Leoben, Austria

${ }^{b}$ Department of Materials Science and Engineering, Shahid Bahonar University of Kerman, Postal code 7618868366, Kerman, Iran

\begin{tabular}{|c|c|}
\hline $\begin{array}{l}\text { ART I C LE INFO } \\
\end{array}$ & A B S T R A C T \\
\hline $\begin{array}{l}\text { Article history: } \\
\text { Received } 6 \text { January, } 2015 \\
\text { Accepted } 15 \text { April } 2015 \\
\text { Available online } \\
17 \text { April } 2015 \\
\text { Keywords: } \\
\text { Thermal modeling } \\
\text { Laser forming } \\
\text { Finite element } \\
\text { Bimodal beam }\end{array}$ & $\begin{array}{l}\text { An analytical and numerical solution is developed for a transient heat conduction equation in } \\
\text { which a plane slab is heated by a bimodal distribution beam over the upper surface. In laser } \\
\text { heat treatment of steel few methods are used to produce a wider and nearly uniform average } \\
\text { irradiance profile. This may be achieved by a bimodal (TEM11) shaped laser beam. In this } \\
\text { paper, Green function method is employed to derive an analytical solution for thermal field } \\
\text { distribution induced by laser forming process. Then 3-D finite element modeling of a slab in } \\
\text { the ANSYS code is used to model the thermal field of laser forming with bimodal beam } \\
\text { distribution. The results show that bimodal beam is useful for obtaining a uniform heat intensity } \\
\text { distribution. }\end{array}$ \\
\hline
\end{tabular}

(C) 2015 Growing Science Ltd. All rights reserved.

\section{Introduction}

Laser forming is a novel technique, where a laser beam causes thermal expansion locally, and deformation is achieved by scanning the laser beam across one side of the material. In laser heat treatment of steel, bimodal distribution beam (Fig. 1) is used to produce a wider and nearly uniform average irradiance profile. The temperature gradients that are developed through the material induce distortion because the temperature is changed with thickness and thus causes different expansion of adjoining layers. Laser forming is currently used because of its technical benefits such as no need to external forces, reduction in cost and increase in flexibility. In past years, considerable attentions have been paid to the comprehending the laser forming mechanisms and the investigation of the effects of laser forming parameters on the deformed shape and mechanical properties of the formed parts (Vollertsen, 1994; Holzer et al., 1994; Shi et al. 2006). In recent years, considerable research are still performed on computer modeling of the laser forming process of plates (Shen et al., 2006; Liu et al., 2007; Yao et al. 2007). In this paper the thermal field distribution of a bimodal laser forming is investigated and compared theoretically and numerically and the good agreements of both methods is demonstrated.

* Corresponding author. Tel.: +43 38424592243

E-mail addresses: masoud.sistaninia@mcl.at (M. Sistaninia) 


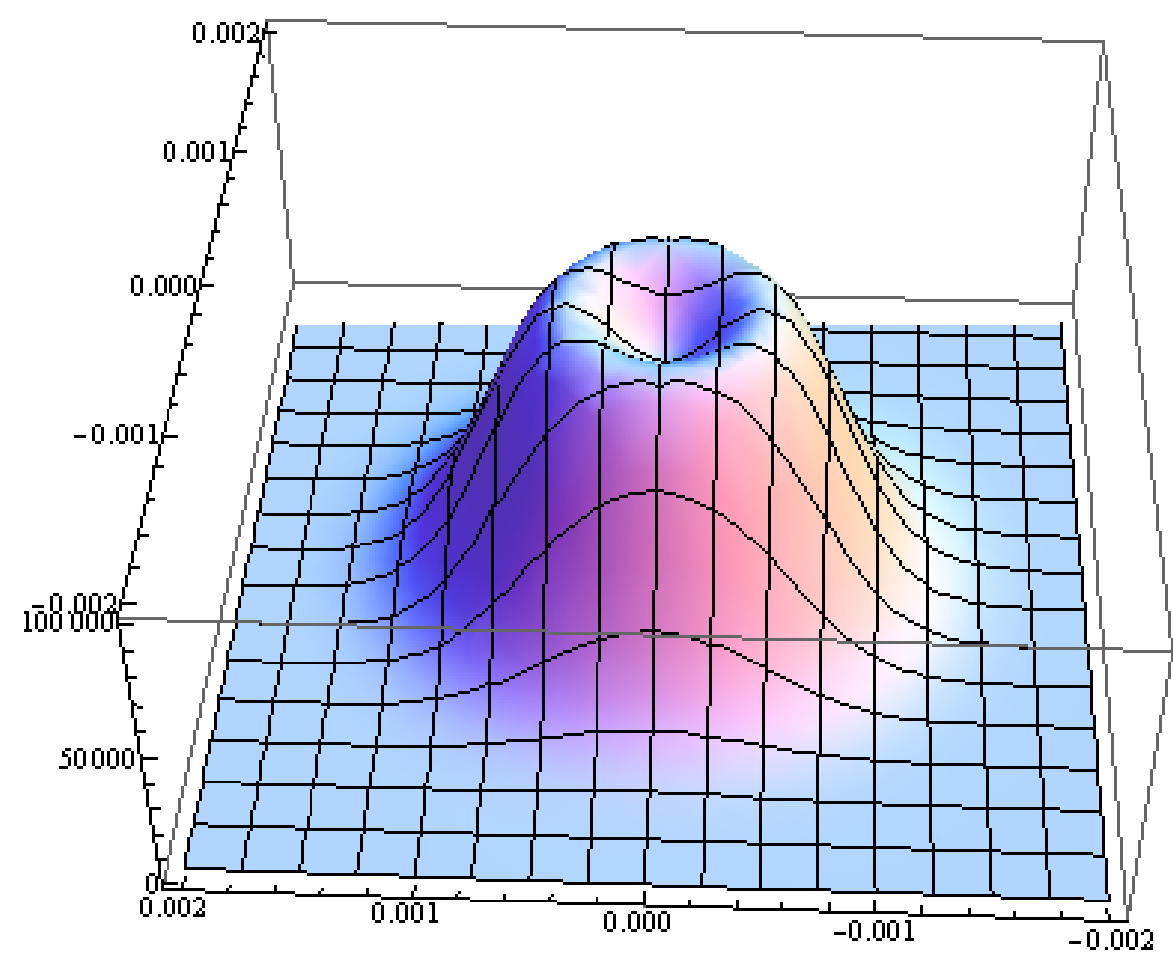

Fig. 1. Schematic of bimodal distribution beam

\section{Analytical model}

An analytical model, based on the renowned transient heat conduction equation, was used to establish the temperature rise as a function of time and step $T(r, t)$ in the material under the action of a laser beam:

$$
\frac{1}{\alpha} \frac{\partial T}{\partial t}-\nabla^{2} T=\frac{f(r, t)}{K},
$$

where $\alpha=K / \rho c_{p}$ is the thermal diffusivity ( $\rho=$ the density, $K=$ thermal conductivity and $c_{p}=$ specific heat) of the material. The internal heat generation term $f(r, t)$, at the right side of Eq. (1), is identified as the energy distribution of the laser beam.

In the case of three-dimensional transient, nonhomogeneous heat conduction problem (given by Eq. (1)), the solution for $T(\mathrm{r}, \mathrm{t})$ is expressed in terms of the three-dimensional Green's function (Polyanin, 2001):

$$
T(r, t)=\frac{\alpha}{K} \int_{\tau=0}^{t} d \tau \int_{R} G\left(\mathbf{r}, t \mid \mathbf{r}^{\prime}, \tau\right) f\left(\mathbf{r}^{\prime}, \tau\right) d \boldsymbol{v}^{\prime}+\left.\int_{R} G\left(\mathbf{r}, t \mid \mathbf{r}^{\prime}, \tau\right)\right|_{\tau=0} F\left(\mathbf{r}^{\prime}\right) d \boldsymbol{v}^{\prime},
$$

where $F\left(\mathbf{r}^{\prime}\right)$ is the initial temperature distribution.

The three-dimensional Green's function can be obtained from the product of the three onedimensional Green's function in rectangular coordinates:

$$
G\left(x, y, z, t \mid x^{\prime}, y^{\prime}, z^{\prime}, \tau\right)=G 1\left(x, t \mid x^{\prime}, \tau\right) \cdot G 2\left(y, t \mid y^{\prime}, \tau\right) \cdot G 3\left(z, t \mid z^{\prime}, \tau\right),
$$

where each of the one-dimensional Green's functions $G 1, G 2$ and $G 3$ depends on the extent of the region (i.e., finite, semi infinite, or infinite) and the boundary conditions. The one-dimensional infinite medium Green's function is obtained as (Polyanin, 2001): 


$$
G\left(x, t \mid x^{\prime}, \tau\right)=[4 \pi \alpha(t-\tau)]^{-1 / 2} \exp \left(-\frac{\left(x-x^{\prime}\right)^{2}}{4 \alpha(t-\tau)}\right)
$$

and the semi infinite medium Green's function when the boundary at $\mathrm{z}=0$ is of the second kind (insulate) is obtained as (Polyanin, 2001):

$$
G\left(z, t \mid z^{\prime}, \tau\right)=[4 \pi \alpha(t-\tau)]^{-1 / 2}\left[\exp \left(-\frac{\left(z-z^{\prime}\right)^{2}}{4 \alpha(t-\tau)}\right)+\exp \left(\frac{\left(z+z^{\prime}\right)^{2}}{4 \alpha(t-\tau)}\right)\right]
$$

Substituting $G 1\left(x, t \mid x\right.$ ',t ) , G2( $\left.y, t \mid y^{\prime}, \mathrm{t}\right)$ and $G 3\left(z, t \mid z^{\prime}, \mathrm{t}\right)$, in Eq. (3) yields the three-dimensional Green's function, for $z^{\prime}=0$,

$$
G\left(x, y, z, t \mid x^{\prime}, y^{\prime}, z^{\prime}=0, \tau\right)=2[4 \pi \alpha(t-\tau)]^{-3 / 2} \exp \left(-\frac{\left(x-x^{\prime}\right)^{2}+\left(y-y^{\prime}\right)^{2}+z^{2}}{4 \alpha(t-\tau)}\right)
$$

Substituting $x^{\prime}=x-r \cos \theta, y^{\prime}=y-r \sin \theta$ and $G\left(\mathbf{r}, t \mid \mathbf{r}^{\prime}, \mathrm{t}\right)$ from Eq. (6) into Eq. (2) the temperature distribution is obtained as:

$$
T(x, y, z, t)=T_{o}+\frac{\alpha}{K} \int_{0}^{t} \int_{-\pi}^{\pi} \int_{0}^{\infty} f(x-r \cos \theta, y-r \sin \theta, \tau) \times 2[4 \pi \alpha(t-\tau)]^{-3 / 2} \exp \left(-\frac{r^{2}+z^{2}}{4 \alpha(t-\tau)}\right) \cdot r d r d \theta d \tau \text {. }
$$

If a piece of finite depth, $L$, is considered the three-dimensional Green's function for a slab with finite depth becomes:

$$
\begin{aligned}
G\left(x, y, z, t \mid x^{\prime}, y^{\prime}, z^{\prime}=0, \tau\right)= & {[4 \pi \alpha(t-\tau)]^{-1} \exp \left(-\frac{\left(x-x^{\prime}\right)^{2}+\left(y-y^{\prime}\right)^{2}}{4 \alpha(t-\tau)}\right) } \\
& \times\left(\frac{1}{L}+\frac{2}{L} \sum_{n=1}^{\infty} e^{-\alpha\left(\frac{n \pi}{L}\right)^{2}(t-\tau)} \cos \left(\frac{n \pi}{L} z\right)\right)
\end{aligned}
$$

and from Eq. (2) with the substitution $x^{\prime}=x-r \cos \theta, y^{\prime}=y-r \sin \theta$, the temperature distribution for a piece of finite depth, $L$, can be expanded as:

$$
\begin{gathered}
T(x, y, z, t)=T_{o}+\frac{\alpha}{K} \int_{0}^{t} \int_{-\pi}^{\pi} \int_{0}^{\infty} \\
f(x-r \cos \theta, y-r \sin \theta, \tau) \times[4 \pi \alpha(t-\tau)]^{-1} \exp \left(-\frac{r^{2}}{4 \alpha(t-\tau)}\right) \\
\times\left(\frac{1}{L}+\frac{2}{L} \sum_{n=1}^{\infty} e^{-\alpha\left(\frac{n \pi}{L}\right)^{2}(t-\tau)} \cos \left(\frac{n \pi}{L} z\right)\right) r d r d \theta d \tau
\end{gathered}
$$

In Eq. (7) and Eq. (|9), $f(x-r \cos \theta, y-r \sin \theta, \tau)$ is the heat intensity distribution.

For a bimodal distribution on a circular disc heat source; the heat release intensity $q(r)$ is a function of $r$. The bimodal distribution which the distance from the center of the distribution curve to one of its peaks on the $r_{i}$-axis is $0.24 r_{o}$ can be expressed by the following equation,

$$
q(r)=4.3677 \frac{Q}{\pi r_{o}^{2}}\left[e^{-\left(3.947\left(r / r_{o}\right)-0.947\right)^{2}}+e^{-\left(3.947\left(r / r_{o}\right)+0.947\right)^{2}}\right]
$$


So, for bimodal distribution that moves along a straight line the function $f(x-r \cos \theta, y-r \sin \theta, \tau)$ takes the form,

$$
\begin{aligned}
& f(x-r \cos \theta, y-r \sin \theta, \tau)= \\
& 4.3677 \frac{Q}{\pi r_{o}^{2}}\left[e^{-\left(\frac{3.947 \sqrt{(x-r \cos \theta-v \tau)^{2}+(y-r \sin \theta)^{2}}}{r_{o}}-0.947\right)^{2}}+e^{-\left(\frac{3.947 \sqrt{(x-r \cos \theta-v \tau)^{2}+(y-r \sin \theta)^{2}}}{r_{o}}+0.947\right)^{2}}\right]
\end{aligned}
$$

\section{Finite element model}

The basic heat-transfer equation considered is:

$\mathrm{F}=\nabla(-K T)$

which relates the heat flux $\mathbf{F}$ to thermal gradient

$$
\nabla(K \nabla T)-\rho c_{p} \frac{\partial T}{\partial t}=-G
$$

This equation applies to unsteady-state heat conduction, where again $\rho$ is the density, $c p$ : the specific heat, $T$ : the temperature, $t$ : the time , $K$ : the thermal conductivity and $G$ : the internal generation of heat. $G$ is zero where the incident laser power is modeled as a heat flux.

In this model the time during which the laser beam continuous irradiation is divided to $n$ increments $\Delta t$, and during each increment $\Delta t$, it can be assumed that the laser beam does not move. The created program determines the thermal load for each node on the heated surface which depends on the laser beam position, heat intensity distribution and the node position. Laser beam position depends on its moving path, speed and time thus the value of load for every node on the heated surface is a function of time and place.

In each increment an implicit 3-D finite element model is used to compute the solution of the heat transfer of Eq. (12). The result of each increment was used as the initial condition of the next time increment. For small $\Delta t$, one can assume that the laser moves continuously. The flow chart of the finite element simulation is shown in Fig. 2.

In this model, it is necessary to make a decision about the element size and shape, time increment and number of step $\mathrm{n}^{*}$ for each increment with the help of the analytical model. Since these quantities are not independent, the following relation can be written:

$$
\left\{\begin{array}{c}
F_{o}=\frac{\Delta x^{2}}{\alpha \Delta t^{*}} \\
\Delta t^{*}=\frac{\Delta t}{n^{*}}
\end{array}\right\}
$$

The Fourier number Fo, which includes material thermo-diffused efficiency $\alpha$, time step $\Delta \mathrm{t}^{*}$ and node spacing $\Delta x$ should be lesser than 2 . For small time increment every load step can be solved by one sub step $\left(\mathrm{n}^{*}=1\right.$ or $\left.\Delta \mathrm{t}=\Delta \mathrm{t}^{*}\right)$. 


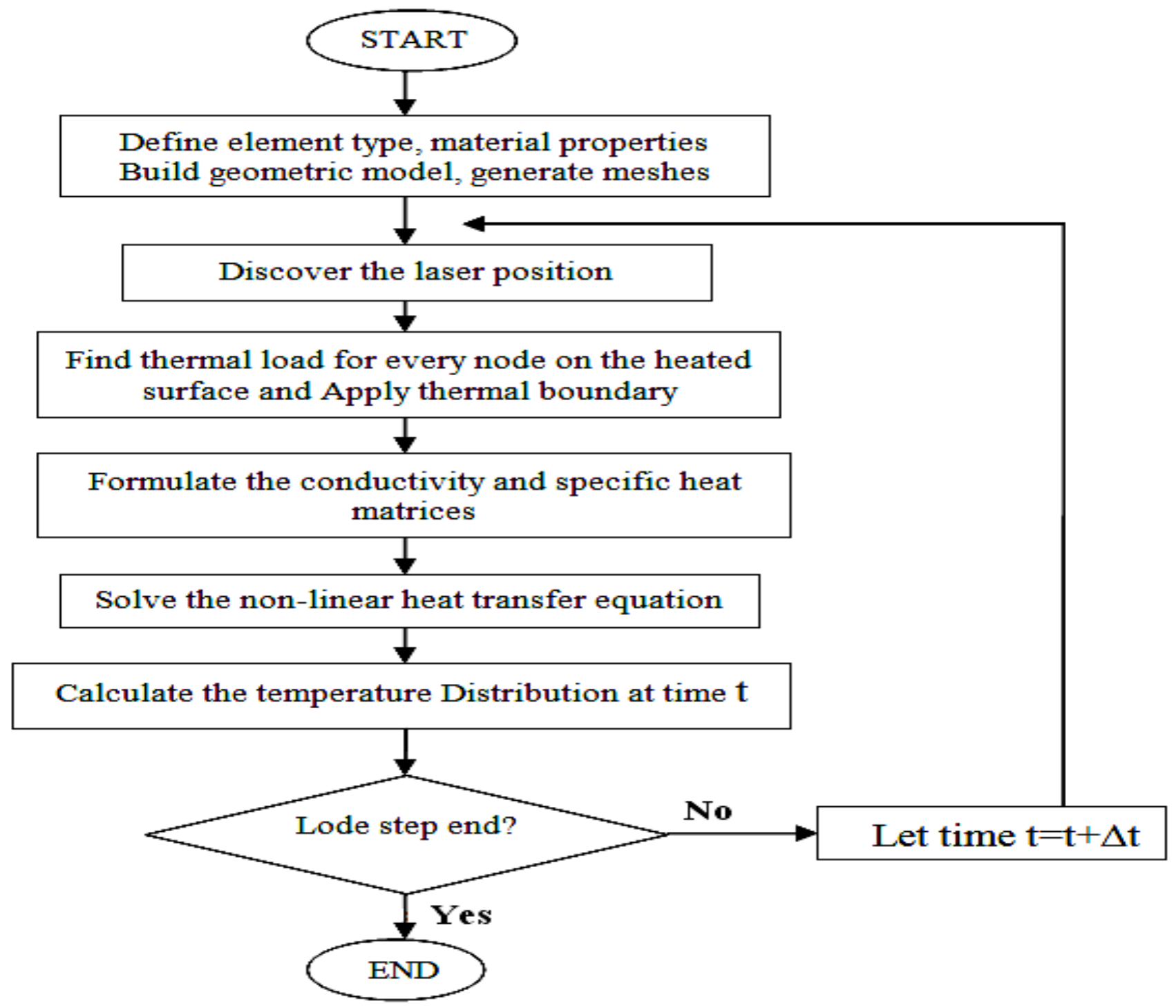

Fig. 2. Flow chart of thermal modeling during laser hardening using a finite element method

\section{Results and discussion}

The 3-D models developed in Fig. 3, is a typical example for investigating the laser forming of a stainless steel plate (AISI 304L). A slab of $30 \times 30 \mathrm{~mm}$ and $4 \mathrm{~mm}$ thickness was used for this investigation. A summary of the processing parameters which are used in this investigation is given in Table 1 . The material was assumed to be homogenous and isotropic. The ambient temperature was set at $25{ }^{\circ} \mathrm{C}$. 


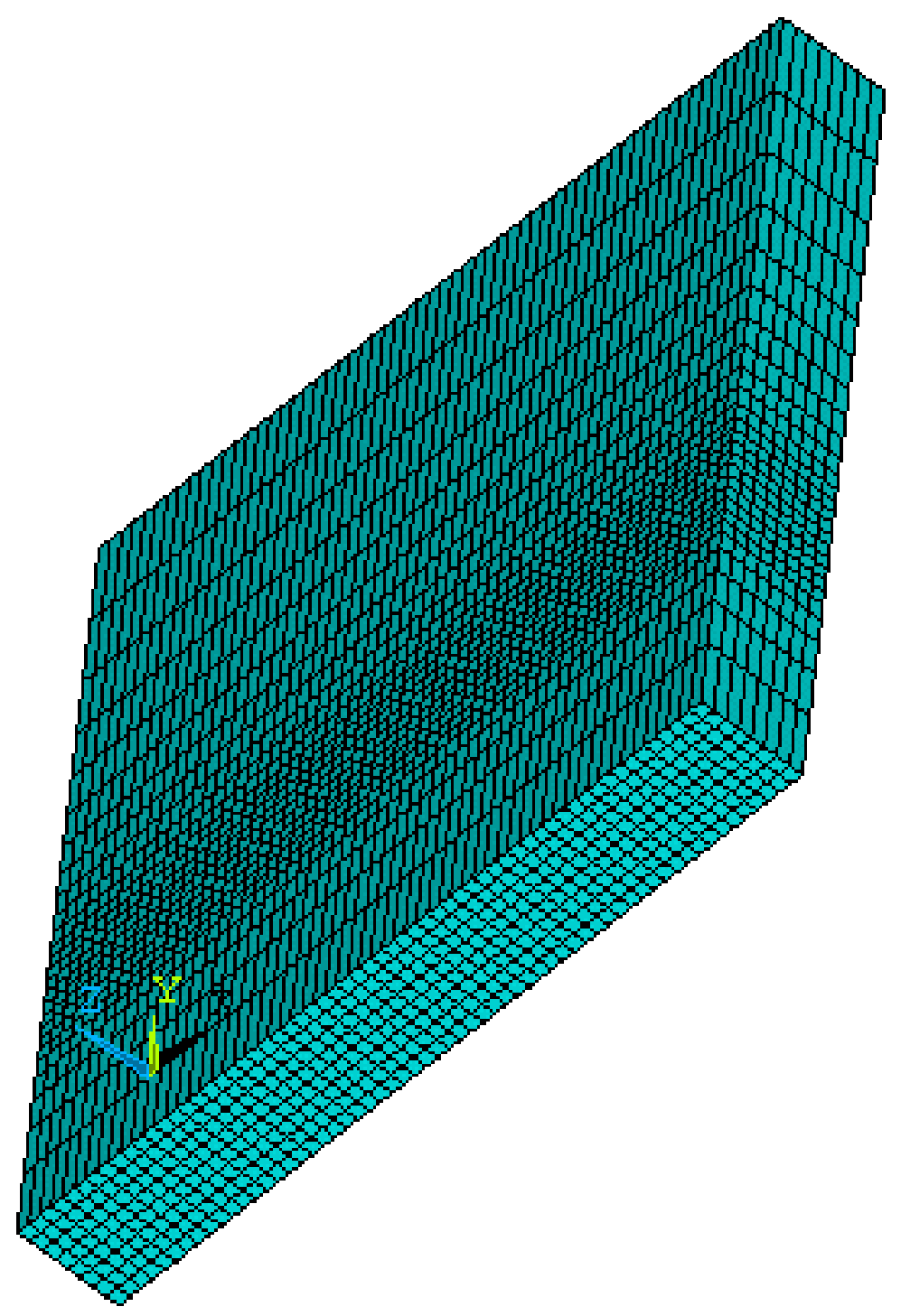

Fig. 3. The 3D model that has been discretized by cubic elements

Table 1. Laser forming parameters

\begin{tabular}{ll}
\hline Laser power, $q(\mathrm{w})$ & 278 \\
Beam diameter, $d(\mathrm{~mm})$ & 4 \\
Scan speed $\left(\mathrm{mm} \cdot \mathrm{sec}^{-1}\right)$ & 30 \\
Absorptivity & 0.72 \\
Thermal conductivity, $\mathrm{k}\left(\mathrm{Wm}^{-1} \mathrm{~K}^{-1}\right)$ & 20 \\
Heat capacity $c\left(\mathrm{~J} \mathrm{~kg}^{-1} \mathrm{~K}^{-1}\right)$ & 552 \\
Thermal diffusivity $\alpha\left(\mathrm{m}^{2} \mathrm{~s}^{-1}\right)$ & $4.61 \mathrm{e}-6$ \\
\hline
\end{tabular}

Fig. 4 shows a comparison between analytical and FEM results for different mesh sizes. This figure shows the temperature history of a point on the heated surface at $x=3.1 \mathrm{~mm}, y=0.48 \mathrm{~mm}$ during laser forming with a bimodal beam. In this comparison the model has been discretized by cubic elements, in order to improve efficiency and reduce calculation dense meshes are used around the heated region. Mesh 1 relates to a meshing which the distance between nodes on the heated surface and around the heated region is $0.25 \mathrm{~mm}$. This mesh under the laser is such that it has sufficient number of elements to capture the inflection of the bimodal distribution (Fig. 3). The number of elements for this mesh is 
84906. As it is obvious for this case we found a good agreement between FEM result and analytical result. Mesh 2 relates to a meshing which the distance between nodes on the heated surface and around the heated region is $0.5 \mathrm{~mm}$ and 8 elements were used along the z-direction (in thickness direction) and mesh 3 relates to a meshing which the distance between nodes on the heated surface and around the heated region again is $0.5 \mathrm{~mm}$ but 4 elements were used along the z-direction (in thickness direction).

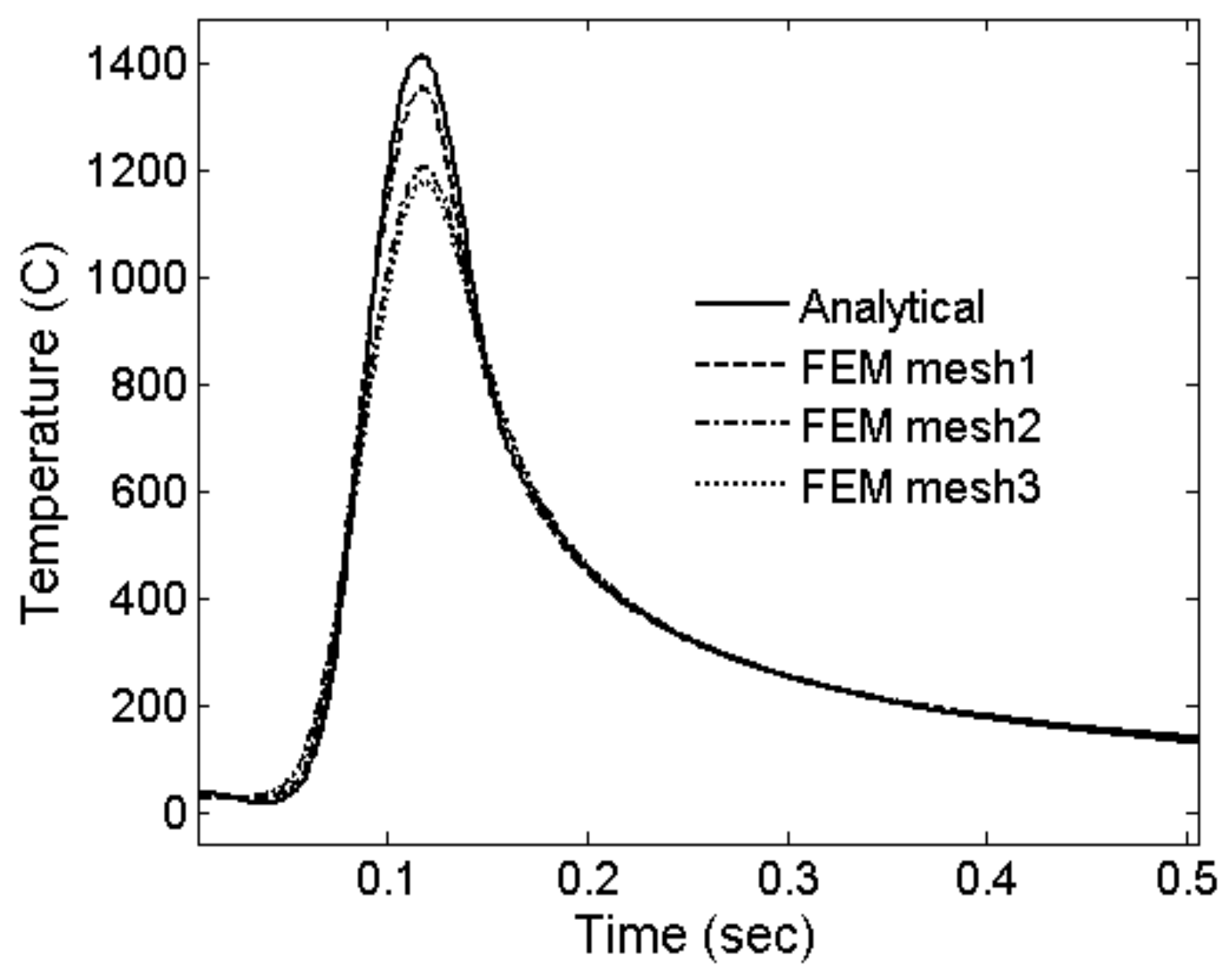

Fig. 4. Comparison of 3-D finite element model and analytical model results for laser forming with bimodal distribution

The mesh 1 is in a good agreement with the analytical model although there is few difference between the analytical and numerical model but if the number of the meshes increase the error dose not decrease considerably and the time of solve increase drastically. Fig. 5 shows the temperature change with time at various points on the heated surface for the bimodal beam model. This Figure shows the maximum temperature occurs on the center line and as the distance increase from the center line the temperature falls precipitously. 


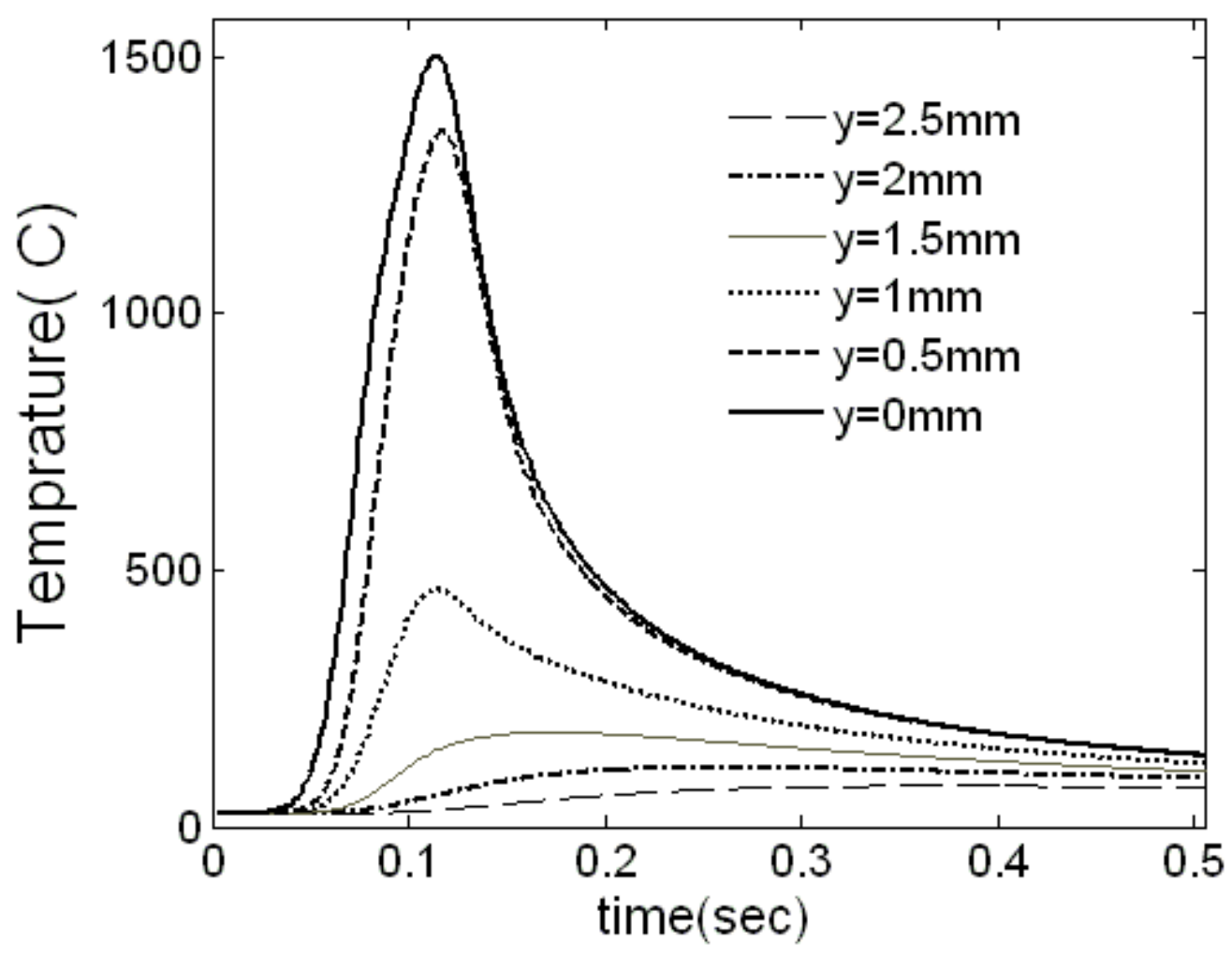

Fig. 5. Temperature history at various points on the heated surface

\section{Conclusions}

The thermal fields in laser forming with bimodal beam were modeled. Green function method has been employed to derive an analytical solution and also a FEM model has been developed to simulate the body temperature. The FEM results are in reasonable agreement with analytical results. The results can be used to design a laser forming process using bimodal heat distributions. The result can be also used for prediction of process parameters (Komanduri \& Hou, 2004).

\section{References}

Holzer, S., Arnet, H., \& Geiger, M. (1994). Extending laser bending for the generation of convex shapes. Proceedings of LANE'94, Section B, 1, Meisenbach Bamberg, Germany, pp. 379-386.

Komanduri, R., \& Hou, Z. B. (2004). Thermal analysis of laser surface transformation hardeningoptimization of process parameters. International Journal of Machine Tools and Manufacture, 44(9), 991-1008.

Liu, F. R., Chan, K. C., \& Tang, C. Y. (2007). Numerical simulation of laser forming of aluminum matrix composites with different volume fractions of reinforcement. Materials Science and Engineering: A, 458(1), 48-57.

Polyanin, A. D. (2001). Handbook of linear partial differential equations for engineers and scientists. CRC press.

Shen, H., Shi, Y., \& Yao, Z. (2006). Numerical simulation of the laser forming of plates using two simultaneous scans. Computational Materials Science, 37(3), 239-245.

Shi, Y., Yao, Z., Shen, H., \& Hu, J. (2006). Research on the mechanisms of laser forming for the metal plate. International Journal of Machine Tools and Manufacture, 46(12), 1689-1697.

Vollertsen, F., (1994). Mechanism and models for laser forming. Proceedings of LANE'94, Section B, 1, Meisenbach Bamberg, Germany, pp 345-360.

Yao, Z., Shen, H., Shi, Y., \& Hu, J. (2007). Numerical study on laser forming of metal plates with preloads. Computational Materials Science, 40(1), 27-32. 